\title{
Ligation of the Thoracic Duct for the Treatment of Chylothorax in Heart Diseases
}

\author{
Paulo M. Pêgo-Fernandes, Fábio B. Jatene, Clayton Cesar Tokunaga, Danielle Tiemi Simão, \\ Ricardo Beirutty, Eliza Rumiko Iwahashi, Sérgio A Imeida de Oliveira
}

São Paulo, SP - Brazil

\begin{abstract}
In children, chylothorax occurs mainly after cardiac and thoracic surgeries. One of the recommended postsurgery treatments is ligation of the thoracic tract, when all other conservative treatments have failed. We report 4 cases of chylothorax in patients who were successfully treated with this approach, which resulted in a decrease in pleural drainage without recurrent chylothorax.
\end{abstract}

Chylothorax refers to the presence of lymphatic fluid in one or both pleural spaces, secondary to leakage in the thoracic duct or one of its main tributaries ${ }^{1}$. Quinke ${ }^{2}$ first described traumatic chylothorax in 1875 , after rupture of the thoracic duct.

When chylothorax is not treated, the risk of death is high, with rates above $45 \%{ }^{3}$, requiring, in the majority of the cases, aggressive treatment.

According to DeMeester ${ }^{4}$, chylothorax may be according to its cause, either congenital, traumatic, neoplastic, or multiple causes. Among the traumatic causes surgical procedures with a thoracic approach may cause thoracic duct lesions, leading to the development of chylothorax. Postoperative chylothorax occurs in less than $1 \%$ of thoracic surgeries, with a prevalence of $0.5 \%$ to $2 \%{ }^{5}$, and may cause nutritional deficiencies, respiratory system involvement, dehydration, and immunosuppression, making the patient more vulnerable to infections.

Postoperative chylothorax is initially treated conservatively ${ }^{6}$. Treatment consists of extracting chyle from the pleural space, the pericardium, or both, through aspiration or through continuous drainage through a chest tube, electrolytic and fluid replacement, and nutritional support. The method of choice for nutritional support is parenteral nutrition, because oral feeding may stimulate chyle production aggravating the clinical picture ${ }^{7}$.

Instituto do Coração do Hospital das Clínicas - FMUSP.

Mailing address: Paulo M. Pêgo-Fernandes - InCor - Av. Dr. Enéas C. Aguiar,44

- 05403-000 - São Paulo, SP, Brazil - E-mail: paulopego@incor.usp.br
If conservative treatment is not effective, surgical treatment should be considered ${ }^{8}$. Studies indicate that ligation of the thoracic duct and pleurodesis are efficient alternatives for resolution of chylothorax ${ }^{9}$.

Recently, thoracic duct ligation through video-assisted thoracic surgery has been used in patients who did not respond to conservative treatment. Kirby et al ${ }^{11}$ describe this conduct as less painful and with fewer postoperative complications.

Pereira et al ${ }^{12}$ describe the use of lymphatic ligation for the treatment of postoperative chylopericardium. However, in our country, ligation of the thoracic duct for the treatment of postoperative chylothorax is still a rare procedure, and at what point the conservative treatment should be replaced by postsurgical treatment is still controversial. Thus, our study evaluates the initial results of ligation of the thoracic tract in 4 patients with chylothorax unresponsive to conservative treatment.

\section{Case Reports}

Case 1 - A four-year-old-male patient with congenital cardiopathy and cyanosis at birth with a previous history of pulmonary atresia without interventricular communication, single left ventricle, hypoplastic right ventricle with sinusoids, left pulmonary artery stenosis, and coronary artery cavitary fistulas. At 5 days of life, he underwent atrial septotomy with the Rashkind procedure, and after 2 days, he underwent a modified left Blalock Taussig shunt procedure. At 4 years old, he underwent a bidirectional Glenn operation with maintenance of the previous Blalock shunt, evolving, in the postoperative period with low cardiac output maintained with vasoactive drugs. He was discharged on the 5th postoperative day, when minor pleural effusion was suspected. A chest X-ray was indicated.

After chylothorax was diagnosed, pleural drainage was started. Parenteral nutrition and fasting were introduced after 1 week. The patient evolved with significant persistence of pleural drainage. Nineteen days after the initial 
clinical picture, one more drainage procedure and surgical cleansing were performed. On the 49th admission day, due to maintenance of chylothorax, the patient underwent ligation of the thoracic duct through a right antero-lateral thoracotomy. A small amount of chyle, multiple pleuro-pulmonary adherences, and a bulge in the pericardial sac were found.

After precise identification of the thoracic duct, ligation was carried out, pericardiocentesis was performed, and $100 \mathrm{~mL}$ of chyle was drained under pressure. Drains were maintained in the 6 th intercostal space. The patient evolved well, with withdrawal of right and left drains on the 16th and 18th postoperative days, respectively.

Case 2 - A one-year-old female patient presenting with a history of viral myocarditis (positive test for Cox sackie virus) evolving to dilated myocardiopathy with congestive heart failure and acute renal failure. She was sent to our service to stabilize the clinical picture and to discuss the possibility of cardiac transplantation. After 1 month, a thoracic X-ray showed an image suggestive of left pleural effusion from a posterior puncture, with leakage of a milky liquid, which was diagnosed through biochemical analysis as chyle. We suspect that the cause of chylothorax was the lesion in the thoracic duct during the insertion of an intracatheter days before. Treatment was initiated with placement of a drain in the left side and fasting maintenance and administration of parenteral nutrition.

After 19 days, due to the persistence of increased pleural drainage and the poor condition of the patient, with great weight loss, the thoracic duct was identified and ligated (fig. 1) through a postero-lateral thoracotomy in the 7th right intercostal space. The patient evolved well, with a decrease in pleural drainage. Oral food consumption was initiated with maintenance of parenteral nutrition until the 8 th postoperative day. The patient showed progressive clinical improvement and underwent cardiac transplantation 25 days after the thoracic duct ligation.

Case 3-A 2-year-old male patient underwent surgical closure of patent ductus arteriosus, evolving with pleural drainage above normal limits. As of the 4 th postoperative day, the appearance of the pleural drainage fluid, which remained elevated, became milkier and was sent for biochemical analysis, and the diagnosis of chylothorax was made. The patient was treated for 5 days with a fat-restricted oral diet; however, the amount of liquid drained did not decrease. Fasting and parenteral nutrition were used for 10 days, with persistent significant drainage and significant weight loss.

Due to the persistence of chylothorax, we opted for ligation of the thoracic duct through a video-assisted thoracic surgery. The patient evolved well with drain withdrawal on the 8 th postoperative day.

Case 4 - A 70-year-old male patient underwent myocardial revascularization. On the $3 \mathrm{rd}$ postoperative day, he evolved with leakage of a milky secretion through the left pleural drain, which later revealed high levels of triglycerides. On the 7th postoperative day, parenteral nutrition was introduced.(BNJ5). On the 14th postoperative, the patient
Ligation of the thoracic duct for the treatment of chylothorax

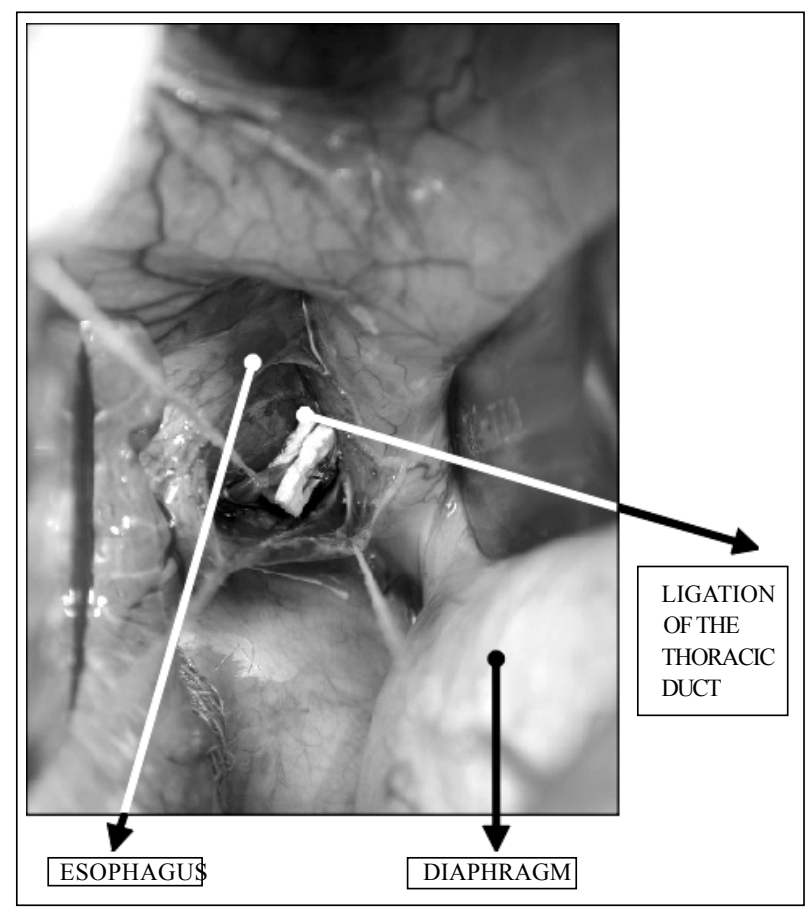

Fig. 1 - Ligation of the thoracic duct.

underwent ligation of the thoracic duct through a right thoracoscopy. Left and right pleural drainage was maintained. The right drain was removed 3 days after ligation. On the 19th postoperative day after revascularization, a liquid diet was reintroduced. Parenteral nutrition was interrupted on the 21 st postoperative day and a light nonfat enteral diet was established, and complemented with medium-chain triglycerides.

On the 24th postoperative day, the patient underwent a chest X-ray that demonstrated bilateral pleural effusion with septation, more evident in the left side, and discreet in the right side. After 4 days, the patient underwent a left thoracotomy with pleural decortication, pleurocentesis, with placement of anterior and posterior drains. A pleural fluid culture indicated the presence of Pseudomonas aeruginosa. On the 5 th postoperative day (33 days after revascularization), a general oral diet was instituted. On the 7th postoperative day, the drains were withdrawn. The patient was discharged on the 9 th postoperative day ( 37 days after revascularization).

\section{Discussion}

Chylothorax is characterized by the effusion of lymphatic fluid in the pleural space. Among other causes, it may originate as a secondary complication of surgical interventions ${ }^{13}$, especially in thoracic approaches, when it is called postoperative chylothorax.

In the 3 pediatric patients in this study, the chylothorax was due to surgical procedures, 2 of them directly related to the cardiac approach and one possibly to the placement of an intracatheter. These data are similar to other studies that 
show that in pediatric patients, postoperative chylothorax occurs, mainly as a thoracic and cardiac postoperative complication ${ }^{14}$.

The 70-year-old patient developed chylothorax after myocardial revascularization with the internal thoracic artery, a rare complication of this procedure due to the location of the thoracic duct ${ }^{15}$.

Postoperative chylothorax may develop in 2 to 4 weeks after surgery and can be mild or severe, according to the volume and the amount of chyle loss ${ }^{14}$. Although it is a rare condition, when not treated, postoperative chylothorax has a high mortality rate, reaching $50 \%{ }^{6}$.

Leakage of chyle in the pleural cavity may compress the ipsilateral lung and, when the secretion is intense, it can cause mediastinum elevation involving the other lung and cardiac function ${ }^{16}$. Additionally, the loss of chyle causes a decrease in the number of $\mathrm{T}$ lymphocytes, involving the immunologic system and predisposing patients to infections ${ }^{5}$. Metabolic alterations are due to depletion of electrolytes, and chylomicrons with long-chain fatty acids determine the metabolic alterations.

Most patients with chylothorax experience dyspnea and pleural secretion. Diagnosis may be confirmed by biochemical and microscopic examinations of the drained liquid, checking for the presence of triglycerides and chylomicrons. The cause can be defined by investigation of the thoracic wall, pleura, lungs, intrathoracic lymphonodus, pulmonary lymphatic or thoracic duct ${ }^{13}$.

Chylothorax treatment aims at a) reducing chyle production, b) treating the underlying causes of intrapleural drainage with fluid replacement, and d) obliteration of the pleural space ${ }^{13}$.

Postoperative chylothorax is initially treated conservatively, based on the drainage of the pleural space and nutritional support, with the use of medium-chain triglyceri$\mathrm{des}^{7}$, low-fat oral diet or enteral rest by giving parenteral nutrition ${ }^{17}$. However, the use of medium-chain triglycerides is avoided by some physicians, after the Peitersen and Jacobsen ${ }^{18}$ study demonstrated the substantial increase in triglyceride content in pleural effusion. Additionally, repeated or continuous drainage of the pleural secretion is inefficient and promotes fluid, plasma protein and electrolyte loss.

If conservative treatment fails, it should be suspended and surgery should be performed. The timing of surgical management is controversial. Some studies recommend surgical intervention if drainage lasts for 1 to 3 weeks ${ }^{17}$, or when daily leakage exceeds $200 \mathrm{~mL}$ to $500 \mathrm{~mL}$ per day.

Surgical treatment of chylothorax includes pleurodesis ${ }^{19}$, ligation of the lymphatic vessels, and ligation of the thoracic duct ${ }^{17}$.

Ligation of the thoracic duct, performed for the first time in 1948 by Lampson ${ }^{3}$ was very efficient low mortality rate. Evidence exists that thoracic duct ligation increases collateral lymphatic circulation regardless of the level of duct ligation. Thus, even with the withdrawal of the thoracic duct and the cisterna chyli, adverse effects are not triggered ${ }^{10}$.

Ligation of the thoracic tract through video-assisted thoracic surgery in the treatment of spontaneous and postoperative chylothorax has been widely described in recent years ${ }^{6}$. It is a very efficient procedure, enabling the identification of the thoracic duct and its selective ligation, and can be indicated when conservative treatment fails, although the presence of extensive pleural adhesions may determine the need for open surgery ${ }^{10}$. Another important factor is that video-assisted surgery causes less postoperative pain and decreases the risk of pulmonary dysfunction ${ }^{11}$. According to Fahimi et $\mathrm{al}^{6}$, video-assisted ligation of the thoracic duct is a great alternative for early treatment of persistent postoperative chylothorax, due to the low cost and facility of its use associated with a lower mortality rate.

For the use of video-assisted thoracic surgery in the treatment of chylothorax, Sachs et al ${ }^{20}$ proposed lymphangiography as a useful method of preoperative localization of the leakage site and reported the value of computed tomography as an additional but not essential method. Fahimi et $\mathrm{al}^{6}$ proposed the administration of a cream meal mixed with Sudan black that enables stabilization of the spot of leakage in the majority of cases, with a simple, efficient, and noninvasive method, in comparison with lymphangiography and computed tomography.

According to Fahimi et al ${ }^{6}$, postoperative chylothorax must follow this conduct: (fig. 2) the use of video-assisted thoracic surgery is indicated when daily leakage exceeds $200 \mathrm{~mL}$ after 2 weeks of conservative treatment.

Some studies demonstrate that the ligation of the thoracic duct by using video-assisted thoracic surgery or thoracotomy for the treatment of chylothorax in children may fail due to the anatomic variations of the thoracic duct or due to the impossibility of identifying the leakage sites ${ }^{21}$.

Such difficulty in identifying and isolating structures with the use of video-assisted thoracic surgery is, in part, related to the duration of chylothorax which, if long-lasting, may cause the development of pleural adherences, as mentioned above ${ }^{10}$, hindering the identification of mediastinal structures. In this case, ligation of the thoracic duct may be performed through open surgery as that performed in patients 1 and 2 .

In other conditions, pleurodesis may be associated with ligation of the thoracic duct in the treatment of chylothorax, as in patient 4 in whom, due to an infectious process, chylothorax persisted even after duct ligation.

Thoracic duct ligation is an efficient method to treat chylothorax. Thus, the possibility of prompt surgical intervention is recommended. At one time, shorter periods of conservative treatments, which are often unsuccessful, could minimize cost and damages to the patients, such as weight loss due to a restrictive diet and the pain caused by the permanence of the chylothorax. However, Sieczka and Harvey ${ }^{5}$ reported that the conservative treatment may cause an unnecessary delay aggravating the nutritional condition, causing immunological deficiency and exposing the patient to the complications of central venous access necessity. 


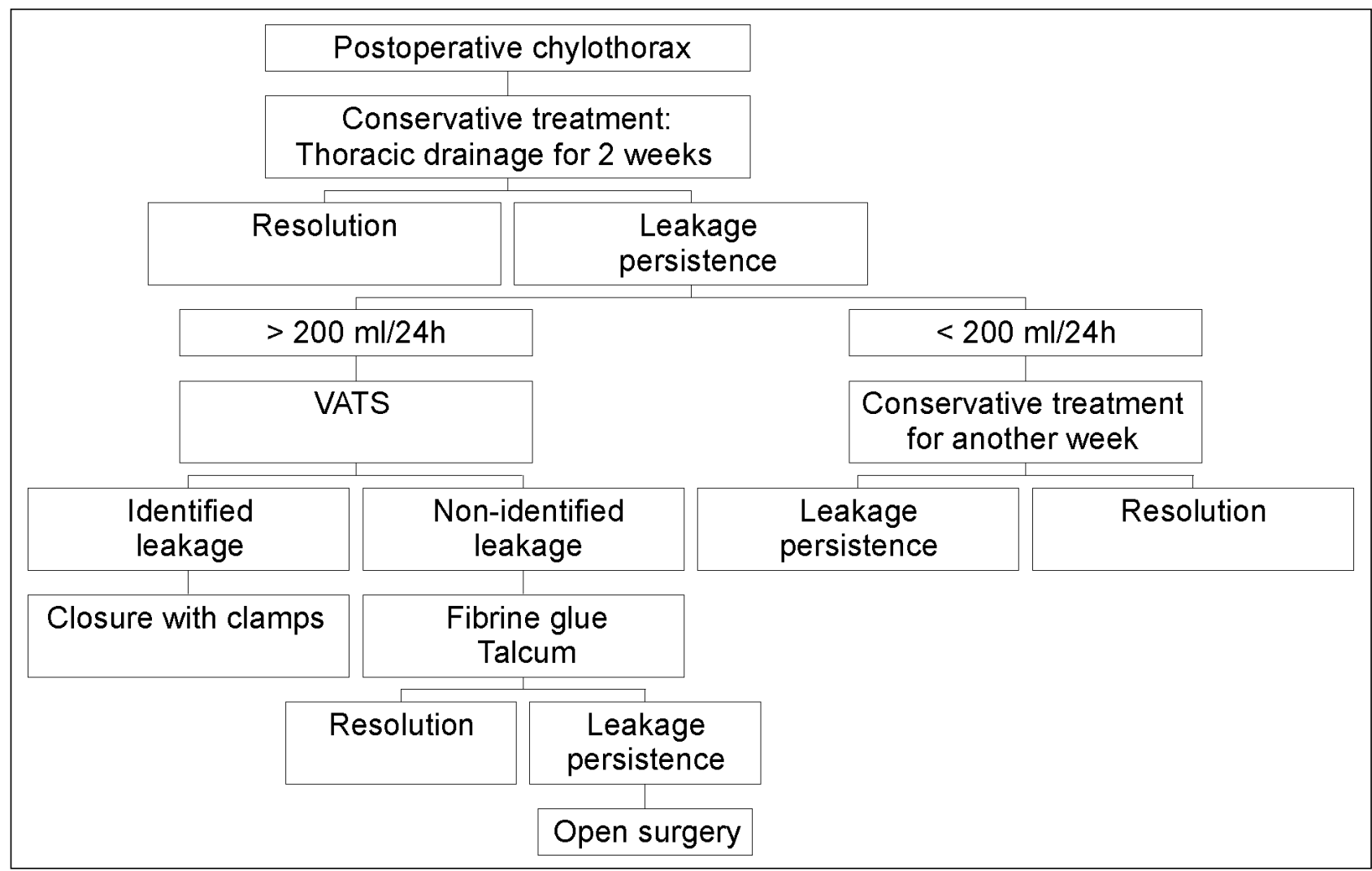

Fig. 2 - Postoperative chylothorax treatment according to Fahimi et al ${ }^{6}$.VATS: video-assisted thoracic surgery.

In conclusion, our 4 patients had significant improvement of in the clinical features of chylothorax after thoracic duct ligation. The treatment was characterized as a good therapeutic alternative for postoperative chylothorax when no response occurs to conservative treatment. Early ligation of the thoracic duct may eliminate the possible failure of conservative treatment, which could be aggressive, causing unnecessary suffering for patients.

\section{References}

1. Miller JI. Chylothorax and anatomy of the thoracic duct. In: Shields TW, ed. General Thoracic Surgery. Philadelphia: Lea \& Febiger, 1989: 625.

2. Wurnig PN, Hollaus PH, et al. Thoracoscopic direct clipping of the thoracic duct for chylopericardium and chylothorax. Ann Thorac Surg 2000; 70: 1662-5.

3. Fahimi H, Casselman FP, et al. Current management of postoperative chylothorax. Ann Thorac Surg 2001; 71: 448-51.

4. Demeester TR. The pleura. In: Sabiston DCaS, EC, ed. Surgery of the Chest. Philadelphia: Lea \& Febiger, 1983.

5. Sieczka EM, Harvey JC. Early thoracic duct ligation for postoperative chylothorax. J Surg Oncol 1996; 61: 56-60.

6. Fahimi H, Casselman FP, Mariani MA, et al. Current management of postoperative chylothorax. Ann Thorac Surg 2001; 71: 448-51.

7. Hashim SA, Roholt HB, Babayan VK, Itallie TB. Treatment of chyluria and chylothorax with medium-chain triglyceride. N Engl J Med 1964; 270: 756-61.

8. Graham DD, McGahren ED, Tribble CG, et al. Use of video-assisted thoracic surgery in the treatment of chylothorax. Ann Thorac Surg 1994; 57: 1507-12.

9. Allen EM, van Heeckeren DW, Spector ML, Blumer JL. Management of nutritio$\mathrm{nal}$ and infectious complications of postoperative chylothorax in children. J Pediatr Surg 1991; 26: 1169-74.

10. Wurnig PN, Hollaus PH, Ohtsuka T, et al. Thoracoscopic direct clipping of the thoracic duct for chylopericardium and chylothorax. Ann Thorac Surg 2000; 70: $1662-5$.

11. Kirby TJ, Mack MJ, Landreneau RJ, Rice TW. Lobectomy-video-assisted thora- cic surgery versus muscle-sparing thoracotomy.: a randomized trial. J Thorac Cardiovasc Surg 1995; 109: 997-1002.

12. Pereira WM, Kalil RAK, Prates PR, Nesralla IA. Cardiac tamponade due to chylopericardium after cardiac surgery. Ann Thorac Surg 1988; 46: 572-3.

13. Browse NL, Allen DR, Wilson NM. Management of chylothorax. Br J Surg 1997; 84: 1711-6.

14. Stringel G, Mercer S, Bass J. Surgical management of persistent postoperative chylothorax in children. Can J Surg 1984; 27: 543-6.

15. Pego-Fernandes PM, Ebaid GX, Nouer GH, et al. Chylothorax after myocardial revascularization with the left internal thoracic artery. Arq Bras Cardiol 1999; 73: 383-90.

16. WolffAB, Silen ML, Kokoska ER, Rodgers BM. Treatment of refractory chylothorax with externalized pleuroperitoneal shunts in children. Ann Thorac Surg 1999; 68: 1053-7.

17. Bond SJ, Guzzetta PC, Snyder ML, Randolph JG. Management of pediatric postoperative chylothorax. Ann Thorac Surg 1993; 56: 469-73.

18. Peitersen B, Jacobsen B. Medium chain triglycerides for treatment of spontaneous, neonatal chylothorax: lipid analysis of the chyle. Acta Paediatr Scand 1977; 66: 121-5.

19. Adler RH, Levinsky L. Persistent chylothorax: treatment by talc pleurodesis. J Thorac Cardiovasc Surg 1978; 76: 859-64.

20. Sachs PB,Zelch MG, Rice TW, et al. Diagnosis and localization of laceration of the thoracic duct: usefulness of lymphangiography and CT. Am J Roentgenol 1991; 157: 703-5.

21. Merrigan BA, Winter DC, O'Sullivan GC. Chylothorax. Br J Surg 1997; 84: 15-20. 